\title{
Using nitrous acid-modified de Man, Rogosa, and Sharpe medium to selectively isolate and culture lactic acid bacteria from dairy foods
}

\author{
Matthew A. Renschler, ${ }^{1} \odot$ Asia Wyatt, ${ }^{1}$ Nnamdi Anene, ${ }^{1}$ Rona Robinson-Hill, ${ }^{1}$ Ethan S. Pickerill, ${ }^{1}$ \\ Nathaniel E. Fox, ${ }^{2}$ Julie A. Griffith, ${ }^{3}$ and John L. McKillip ${ }^{1 *}$ (i) \\ ${ }^{1}$ Department of Biology, Ball State University, Muncie, IN 47306 \\ ${ }^{2}$ Earle A. Chiles Research Institute, Portland, OR 97213 \\ ${ }^{3}$ Department of Physiology and Pharmacology, School of Medicine, West Virginia University, Morgantown 26506
}

\section{ABSTRACT}

Nitrous acid was used to modify traditional de Man, Rogosa, Sharpe medium to evaluate whether the addition of sodium nitrite to MRS medium could improve the rate of growth and density of various lactic acid bacteria and nontarget species. Yogurt and Cheddar cheese were inoculated with individual bacterial species followed by the recovery and enumeration of the species using the pour plate method to compare the sensitivity between nitrous acid-modified MRS (mMRS) and traditional MRS. Lactobacillus delbrueckii ssp. bulgaricus were recovered at significantly higher counts from cheese in nitrous acid mMRS than MRS, whereas no significant difference was observed for other species and food systems. Growth curves were also generated for multiple lactic acid bacteria as well as nonstarters in both mMRS and MRS to measure the selectivity of nitrous acid mMRS. The selectivity evaluation of nitrous acid mMRS demonstrated that 5 of the tested lactic acid bacterial species (Bifidobacterium longum, Streptococcus salivarius, Lactococcus lactis, Lactobacillus acidophilus, and Lactobacillus delbrueckii ssp. bulgaricus) grew to significantly higher densities more rapidly in mMRS broth than in traditional MRS. Nontarget bacteria Enterococcus faecalis and Bacillus cereus revealed a more prolific growth rate and higher optical density readings in traditional MRS compared with mMRS. It was determined that nitrous acid mMRS is a viable alternative medium for culturing selected lactic acid bacteria, and offers an improved formulation of MRS for use in standard evaluation methods and optimization of probiotic and other dairy cultures.

Key words: lactic acid bacteria, de Man, Rogosa, and Sharpe medium, selective recovery

Received May 30, 2019.

Accepted September 11, 2019.

*Corresponding author: jlmckillip@bsu.edu

\section{INTRODUCTION}

Lactic acid bacteria (LAB) are facultatively anaerobic gram-positive bacteria vital as a tool in the dairy industry. Homofermentative LAB include Streptococcus thermophilus and Lactococcus lactis ssp. lactis, whereas heterofermenters include Lactobacillus acidophilus, Lactobacillus delbrueckii ssp. bulgaricus, and Leuconostoc mesenteroides (Sharpe, 1979; Carr et al., 2002). Although all LAB share many fundamental characteristics, each individual group has unique growth conditions including an optimal $\mathrm{pH}$ range. These variable growth preferences impart challenges to using an all-purpose medium for LAB cultivation. For example, Lactobacillus prefers a $\mathrm{pH}$ of below 4.5, whereas Leuconostoc prefers a $\mathrm{pH}$ of 5.5 or greater. A recent study by Gezginc et al. (2015) used MRS agar at $37^{\circ} \mathrm{C}$ for Lactobacillus delbrueckii ssp. bulgaricus and SM17 agar at $42^{\circ} \mathrm{C}$ for $S$. thermophilus. These differences in growth requirements have led to the large array of media used for the cultivation of each specific lactic acid bacterial species.

Combinations of these bacteria, for example $S$. thermophilus and Lactobacillus delbrueckii ssp. bulgaricus, may interact synergistically during and after fermentation (Herve-Jimenez et al., 2009). The most commonly used medium for routine culturing of these LAB is de Man, Rogosa, and Sharpe (MRS; Ao et al., 2012; Gezginc et al., 2015) that can be modified with sorbitol, galactose, or maltose (Dave and Shah, 1996; Oberg et al., 2011). Other media used for isolation of specific LAB include Elliker, Lee's medium for Streptococcus species (Biede et al., 1975), Lactobacillus selective agar, LM17 agar (Gezginc et al., 2015), SM17 agar, and Edward's agar (Sawant et al., 2002). The ability to culture multiple LAB on an improved medium is needed due to increased government regulations (Carr et al., 2002) and to increase hygiene monitoring as phages are well-known contaminants of LAB starter cultures (Collins, 1962; Jarvis, 1989). While MRS medium is 
used today for general culturing of LAB, Davidson and Cronin (1973) developed a nitrous acid-based medium to selectively isolate LAB from yogurt, cheese, or sauerkraut. Nitrous acid with MRS medium may be able to selectively isolate LAB from food samples, with nitrous acid being the selective agent. This nitrous acid-modified MRS medium ( $\mathbf{m M R S}$ ) could increase hygiene monitoring of starter cultures for a variety of LAB by inhibiting growth of nonstarter bacteria such as Bifidobacterium spp. while maintaining the sensitivity of traditional MRS medium. We hypothesize that the use of nitrous acid-modified MRS would offer an improved means of selective isolation and enumeration of LAB common in diary fermentations compared with traditional MRS, while still maintaining desirable selectivity by inhibiting growth of nontarget gram-positive bacteria.

\section{MATERIALS AND METHODS}

\section{Maintenance of Cultures}

Bacterial cultures of Lactobacillus delbrueckii ssp. bulgaricus (Presque Isle Cultures 546, Erie, PA), Lactococcus lactis (Presque Isle Cultures 525A), Lactobacillus acidophilus (Presque Isle Cultures 545), Streptococcus thermophilus ssp. salivarius (Presque Isle Cultures 518), and Bifidobacterium longum ATCC55818 (ATCC, Manassas, VA) were maintained by subculturing weekly into MRS broth (Alpha Biosciences L12-104, Baltimore, MD) and incubating statically at $37^{\circ} \mathrm{C}$. Nontarget gram-positive bacteria (Enterococcus faecalis and Bacillus cereus ATCC14579) were likewise incubated in MRS broth under the same conditions. Bacterial strains used were selected to represent members of each genus/species, with the understanding that minor strain-to-strain variation may exist among these type species as well as natural isolates used in industry.

\section{Modified MRS Agar Preparation}

The MRS agar was prepared by adding agar powder at a concentration of $17 \mathrm{~g} / \mathrm{L}$ to MRS broth (Alpha Biosciences, Baltimore, MD) as prepared by manufacturer's instructions. Modified MRS agar was prepared by adding $1 \mathrm{~mL}$ of filter sterilized $(0.22 \mu \mathrm{m}$ pore size $)$ $6 \% \mathrm{wt} / \mathrm{vol}$ sodium nitrite solution per $100 \mathrm{~mL}$ of medium (Davidson and Cronin, 1973) after the MRS had been autoclaved and tempered to $55^{\circ} \mathrm{C}$ for $30 \mathrm{~min}$. After the addition of sodium nitrite, the $\mathrm{pH}$ of the mMRS agar was adjusted up to $6.5( \pm 0.05)$ using $\mathrm{HCl}$ and maintained at $55^{\circ} \mathrm{C}$ before use.

\section{Confirmation of Bacterial Densities}

All bacterial strains were subcultured by looping into MRS broth (from a fresh overnight suspension of each) and incubating for $30 \mathrm{~min}$ to $2 \mathrm{~h}$ to reach the respective target density. After incubation, the optical density of each bacterial culture was measured to confirm density within the range of 0.05 to 0.09 at $\mathrm{A}_{600}$. Each bacterial culture was serially diluted and pour-plated onto MRS agar to confirm the target density of $10^{5} \mathrm{cfu} / \mathrm{mL}$ for each bacterial species, a value corresponding to mid-log phase.

\section{Evaluation of mMRS Using Yogurt}

Plain unflavored lowfat dairy yogurt (purchased at a local retailer) was used to evaluate mMRS for Lactococcus lactis, Lactobacillus acidophilus, Streptococcus thermophilus ssp. salivarius, and Lactobacillus delbrueckii ssp. bulgaricus. Each bacterial species was subcultured into fresh MRS broth to the desired optical density (OD) corresponding to $10^{5} \mathrm{cfu} / \mathrm{mL}$ as described earlier. Subsequently, $1 \mathrm{~mL}$ of each broth suspension was transferred to a microcentrifuge tube and centrifuged for $3 \mathrm{~min}$ at $9,300 \times g$ at ambient temperature. The supernatant was decanted and the pellet washed with 1 $\mathrm{mL}$ of $0.1 \%$ saline and centrifuged again with the same settings. Most of the supernatant was aspirated with a small amount left to resuspend the pellet. Yogurt was measured (in 9-g aliquots) and placed into sterile plastic containers. Each was inoculated by transferring the re-suspended pellet for each bacterial species into separate yogurt samples and aseptically mixing well. An uninoculated MRS broth was also transferred (1 $\mathrm{mL}$ ) into a yogurt sample to serve as a negative control. The inoculated yogurt samples were incubated for $1 \mathrm{~h}$ at ambient temperature $\left(23^{\circ} \mathrm{C}\right)$.

While the yogurt samples incubated, the mMRS medium was prepared as outlined above. After incubation, yogurt samples were pour-plated onto MRS agar and mMRS agar plates in triplicate for each dilution of each bacterial species for each medium type, essentially as described by Duncan et al. (2004). The negative controls were set up using the 1:100 dilution of the yogurt blank for each medium as well as setting up a pour plate for each medium type with no yogurt added. All plates were incubated at $37^{\circ} \mathrm{C}$ for $48 \mathrm{~h}$ under microaerophilic conditions (using a candle jar) and colonies counted using a colony counter. The Mann-Whitney test was used to compare counts between each medium type.

The mMRS medium was also tested using yogurt that was not artificially contaminated with LAB to 
enumerate naturally occurring bacteria in yogurt. Yogurt was pour plated in triplicate in both MRS and mMRS media as undiluted yogurt (1 g of fat-free plain yogurt per plate) and $10^{-1}$. A negative control for each medium with no yogurt was also made. Plates were incubated microaerophilically at $37^{\circ} \mathrm{C}$ for $48 \mathrm{~h}$ and then counted using a colony counter. A 2-sample $t$-test was used to compare counts between each medium type.

\section{Evaluation of mMRS Using Cheese}

Shredded mild Cheddar cheese was used to evaluate mMRS for recovery of Lactobacillus delbrueckii ssp. bulgaricus. After incubation for approximately $1 \mathrm{~h}$ after subculturing, the OD was measured to confirm bacterial growth to a target density of $10^{5} \mathrm{cfu} / \mathrm{mL}$. After measuring the OD, the bacterial pellet was prepared as previously described. Shredded cheddar cheese was measured out $(2.5 \mathrm{~g})$ and placed into sterile plastic bags with $22.5 \mathrm{~mL}$ of $0.1 \%$ peptone tempered to $50^{\circ} \mathrm{C}$ and stomached momentarily. Cheese was inoculated by transferring the resuspended pellet into separate cheese samples. The blank from earlier was also transferred $(1 \mathrm{~mL})$ into a cheese sample to serve as a negative control. The inoculated cheese samples were incubated for $1 \mathrm{~h}$ at room temperature as previously described. After incubation, cheese samples were diluted and pour plated separately into MRS agar and mMRS agar. Pour plates were set up in triplicate for each dilution of each bacterial species for each medium type. The negative controls were set up using the 1:100 dilution of the cheese blank for each medium as well as setting up a pour plate for each medium type with no cheese added. All plates were incubated at $37^{\circ} \mathrm{C}$ for 48 or 72 $\mathrm{h}$ depending on the size of colonies and then counted using a colony counter. A Mann-Whitney test was used to compare counts between each medium type for each bacterial species. A different bag of shredded Cheddar cheese was used for the last trial of artificially contaminated cheese.

The mMRS medium was also tested using cheese that was not artificially contaminated with LAB to enumerate naturally occurring bacteria in cheese. Cheese samples were set up the same as above (2.5 g of shredded Cheddar into $22.5 \mathrm{~mL}$ of $0.1 \%$ peptone tempered to $50^{\circ} \mathrm{C}$ ) was pour plated in triplicate in both MRS and mMRS media. A negative control for each medium with no cheese was also made. Plates were incubated at $37^{\circ} \mathrm{C}$ for 48 or $72 \mathrm{~h}$ depending on colony size and then counted using a colony counter. A Mann-Whitney test was used to compare counts between each medium type.
The MRS and mMRS separate broth tubes were inoculated in triplicate with each LAB species previously mentioned along with nontargets Enterococcus faecalis and Bacillus cereus.

Optical density was measured for each tube regularly for $18 \mathrm{~h}$; 2 -sample $t$-test was used to compare growth rates $(P=0.05)$. Growth curves constructed for each species in each medium to compare relative growth rates via OD.

\section{Selectivity Evaluation of mMRS}

The selectivity of nitrous acid mMRS was evaluated by comparing OD curves for species between MRS and mMRS broth (rate of growth). Lactococcus lactis, Streptococcus thermophilus ssp. salivarius, Lactobacillus acidophilus, Lactobacillus delbrueckii ssp. bulgaricus, Enterococcus faecalis, Bifidobacterium longum, and Bacillus cereus were each inoculated (loopful) in triplicate into both MRS and mMRS broths. After inoculation, the OD at $600 \mathrm{~nm}$ was measured for each culture at various time points and recorded to construct an OD curve to compare growth rates of each species between MRS and mMRS broth. Enterococcus faecalis, Bifidobacterium longum, and Bacillus cereus were used to represent nonstarters or possible contaminants of starter cultures to determine if nitrous acid mMRS is more selective or inhibits their growth to some degree. A 2-sample $t$-test was used to compare growth between MRS and nitrous acid mMRS after incremental periods of growth.

\section{RESULTS}

\section{mMRS Versus MRS Bacterial Recovery from Yogurt}

Seeded bacteria were allowed to remain in the yogurt (and cheese) for $1 \mathrm{~h}$ to become somewhat associated with the food components (as opposed to immediately plating), and no fermentation was expected or desired from these inoculated bacteria. Three independent experiments were conducted for each evaluation of nitrous acid mMRS and the mean colony-forming units per milliliter were compared between the 2 variations for each species and food system. The Mann-Whitney test was used to compare mean colony-forming units per milliliter as the distributions were not normal except for the natural yogurt that was not artificially contaminated (data not shown). A 2-sample $t$-test was used for these normally distributed data. In the evaluation of nitrous acid mMRS using yogurt, no significant difference was detected between nitrous acid mMRS and traditional MRS in the recovery of viable cell densities for any 
A

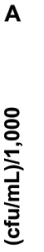

80
70
60
50
40
30
20
10

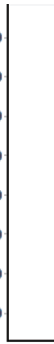

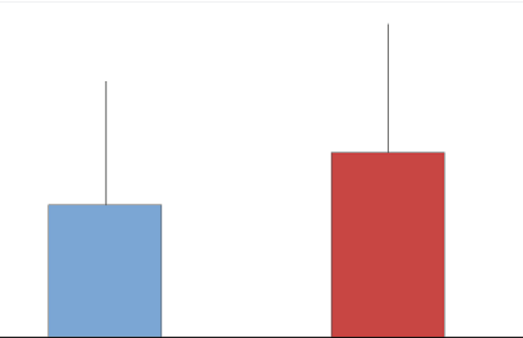

L. lactis, MRS

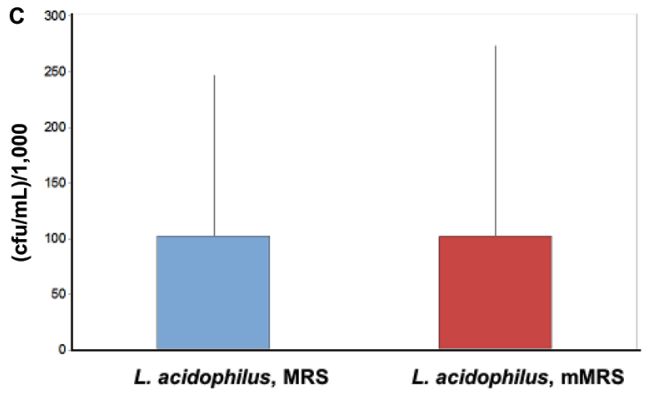

E

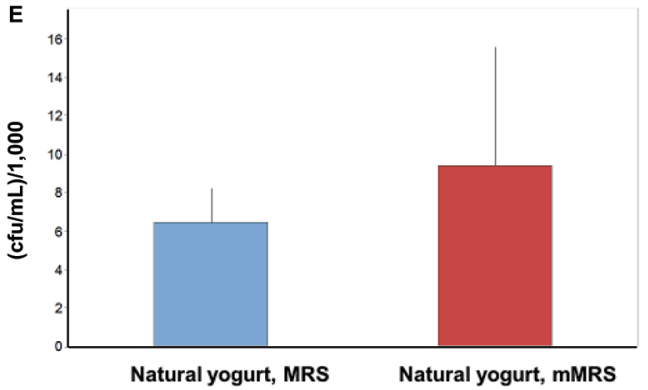

B
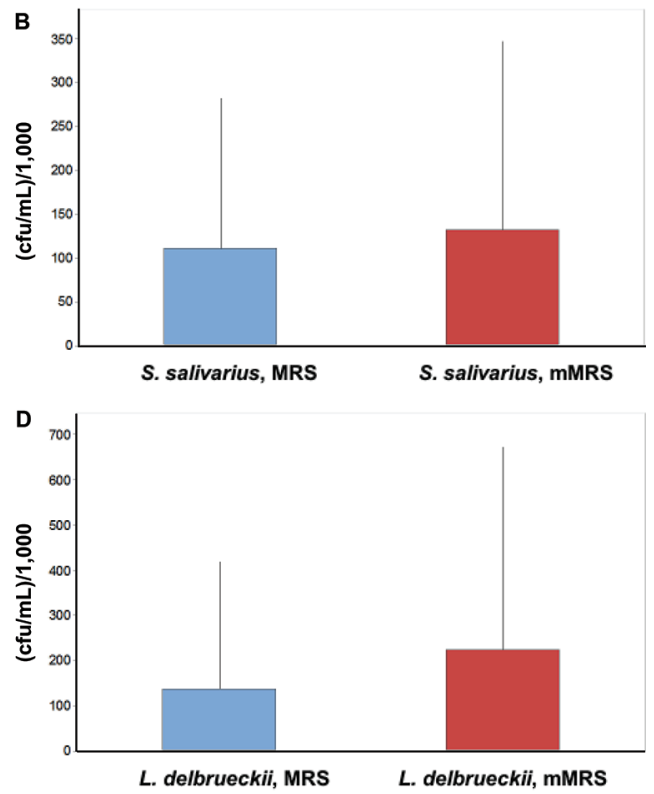

Figure 1. (A) Mean viable cell densities (measured in cfu/mL divided by 1,000 along with error bar of 1 SD) revealed no significant difference in the recovery of Lactococcus lactis from yogurt between de Man, Rogosa, and Sharpe (MRS; mean cfu $/ \mathrm{mL}=3.685 \times 10^{4}$ ) and nitrous acid-modified MRS (mMRS; mean cfu $/ \mathrm{mL}=4.997 \times 10^{4} ; P=0.3172$ ). (B) Mean viable cell densities (measured in cfu/mL divided by 1,000 along with error bar of $1 \mathrm{SD}$ ) showed no significant difference in the recovery of Streptococcus thermophilus ssp. salivarius from yogurt between MRS (mean cfu $/ \mathrm{mL}=1.105 \times 10^{5}$ ) and nitrous acid mMRS (mean cfu $\left./ \mathrm{mL}=1.314 \times 10^{5} ; P>0.99\right)$. (C) Mean viable cell densities (measured in $\mathrm{cfu} / \mathrm{mL}$ divided by 1,000 along with error bar of $1 \mathrm{SD}$ ) showed no significant difference in the recovery of Lactobacillus acidophilus from yogurt between MRS (mean cfu $/ \mathrm{mL}=1.016 \times 10^{5}$ ) and nitrous acid mMRS (mean cfu $/ \mathrm{mL}=1.014 \times 10^{5} ; P=0.8430$ ). (D) Mean viable cell densities (measured in cfu/mL divided by 1,000 along with error bar of $1 \mathrm{SD}$ ) showed no significant difference in the recovery of Lactobacillus delbrueckii ssp. bulgaricus from yogurt between MRS (mean cfu $\left./ \mathrm{mL}=1.351 \times 10^{5}\right)$ and nitrous acid mMRS $\left(\mathrm{mean} \mathrm{cfu} / \mathrm{mL}=2.241 \times 10^{5} ; P=0.4152\right)$. $(\mathrm{E})$ Mean viable cell densities (measured in $\mathrm{cfu} / \mathrm{mL}$ divided by 1,000 along with error bar of $1 \mathrm{SD}$ ) showed no significant difference in the recovery of naturally occurring bacteria from yogurt between MRS (mean cfu $/ \mathrm{mL}=645)$ and nitrous acid $\mathrm{mMRS}(\mathrm{mean} c \mathrm{cu} / \mathrm{mL}=942 ; P=0.207)$.

species. No significant difference $(P=0.3172)$ was observed in the recovery of Lactococcus lactis from yogurt between nitrous acid mMRS (mean $\mathrm{cfu} / \mathrm{mL}=4.997 \times$ $10^{4}$ ) and MRS (mean cfu/mL $=3.685 \times 10^{4} ;$ Figure 1A). Moreover, no significant difference $(P>0.99)$ was evident in the recovery of Streptococcus thermophilus ssp. salivarius from yogurt between nitrous acid mMRS $\left(\right.$ mean $\left.\mathrm{cfu} / \mathrm{mL}=1.314 \times 10^{5}\right)$ and MRS (mean $\mathrm{cfu} /$ $\mathrm{mL}=1.105 \times 10^{5} ;$ Figure 1B). Likewise, no significant difference $(P=0.8430)$ was seen in the recovery of Lactobacillus acidophilus from yogurt between nitrous acid mMRS (mean cfu $/ \mathrm{mL}=1.014 \times 10^{5}$ ) and MRS (mean $\mathrm{cfu} / \mathrm{mL}=1.016 \times 10^{5} ;$ Figure $\left.1 \mathrm{C}\right)$. No significant difference $(P=0.5121)$ was observed in the recovery of
Lactobacillus delbrueckii ssp. bulgaricus from yogurt between nitrous acid mMRS (mean $\mathrm{cfu} / \mathrm{mL}=1.351 \times$ $10^{5}$ ) and nitrous acid mMRS (mean cfu/mL $=2.241 \times$ $10^{5} ; P=0.4152 ;$ Figure 1D). No significant difference $(P=0.207)$ was observed in the recovery of naturally occurring bacteria from yogurt between nitrous acid mMRS (mean $\mathrm{cfu} / \mathrm{mL}=942$ ) and MRS (mean cfu $/ \mathrm{mL}$ $=645$; Figure 1E).

\section{mMRS Versus MRS Bacterial Recovery from Cheddar Cheese}

Nitrous acid mMRS recovered significantly higher counts (mean $\left.\mathrm{cfu} / \mathrm{mL}=1.065 \times 10^{6}, P<0.005\right)$ of 


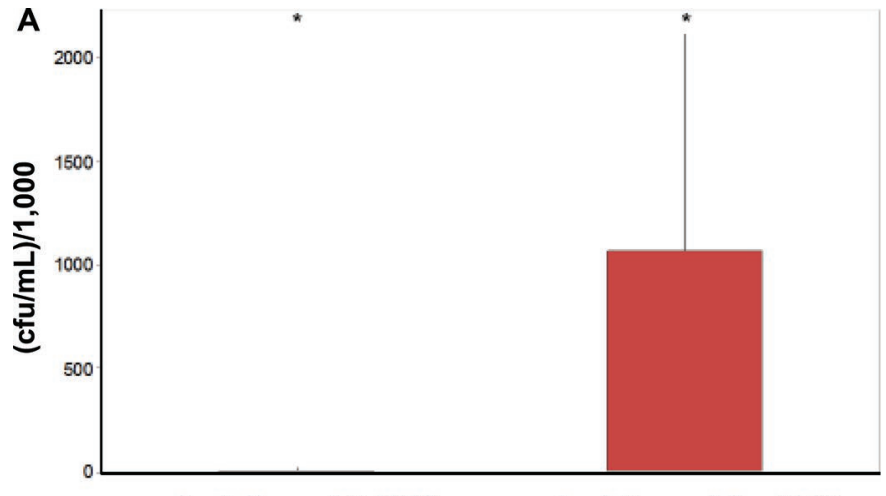

L. delbrueckii, MRS

L. delbrueckii, mMRS

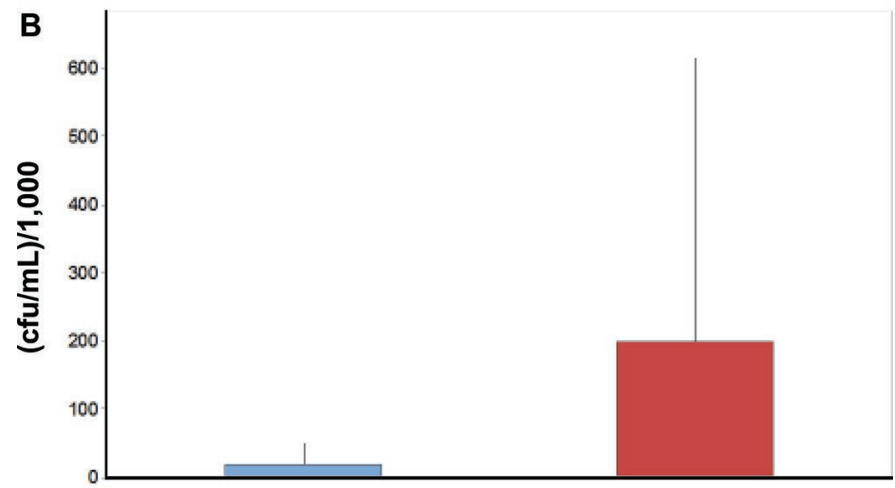

Natural Cheese, MRS

Natural Cheese, mMRS

Figure 2. (A) Mean viable cell densities (measured in $\mathrm{cfu} / \mathrm{mL}$ divided by 1,000 along with error bar of $1 \mathrm{SD}$ ) showed that nitrous acidmodified de Man, Rogosa, and Sharpe (mMRS; mean cfu/mL $=1.065$ $\times 10^{6}$ ) recovered significantly higher counts of Lactobacillus delbrueckii from shredded Cheddar cheese than traditional MRS (mean $\mathrm{cfu} / \mathrm{mL}$ $=7.620 \times 10^{3} ; P<0.005$ ). (B) Mean viable cell densities (measured in $\mathrm{cfu} / \mathrm{mL}$ divided by 1,000 along with error bar of $1 \mathrm{SD}$ ) suggest that nitrous acid mMRS (mean $\mathrm{cfu} / \mathrm{mL}=1.992 \times 10^{5}$ ) recovered higher counts of naturally occurring bacteria from shredded Cheddar cheese than traditional MRS (mean $\left.\mathrm{cfu} / \mathrm{mL}=1.847 \times 10^{4} ; P=0.0621\right) .{ }^{*} P$ $<0.05$.

Lactobacillus delbrueckii ssp. bulgaricus from Cheddar cheese compared with traditional MRS (mean cfu/mL $=7.620 \times 10^{3} ;$ Figure $2 \mathrm{~A}$ ). Our results also revealed that nitrous acid mMRS recovers higher counts (mean $\left.\mathrm{cfu} / \mathrm{mL}=1.992 \times 10^{5}, P=0.0621\right)$ of naturally occurring bacteria from cheese versus traditional MRS $\left(\right.$ mean $\mathrm{cfu} / \mathrm{mL}=1.847 \times 10^{4} ;$ Figure $\left.2 \mathrm{~B}\right)$.

\section{mMRS Versus MRS Lactic Acid Bacterial Growth Rates}

Mean optical density measurements was used for each species in each medium to construct an OD curve to compare growth in nitrous acid mMRS versus standard MRS broth. Lactococcus lactis, Streptococcus salivarius, Bifidobacterium longum, Lactobacillus delbrueckii ssp. bulgaricus, and Lactobacillus acidophilus all grew to sig- nificantly higher densities more rapidly in nitrous acid mMRS broth than in MRS broth $(P<0.005)$ by the end of the 18-h incubation time period (Figures 3A-3E, respectively). No significant difference was observed in the growth of Bacillus cereus ATCC14579 between nitrous acid mMRS and MRS broth $(P>0.05)$. However, nontarget gram-positive Enterococcus faecalis grew to significantly higher densities in traditional MRS broth compared with mMRS $(P<0.05$ at 18 h, Figure 4B).

\section{DISCUSSION}

The goal of this research was to explore the possibility of improving on the existing MRS medium formulation for more rapid cultivation of lactobacilli and other LAB while maintaining or enhancing the selective nature against nontarget gram-positive bacteria (Enterococcus faecalis and Bacillus cereus). Regarding sensitivity of recovery from artificially seeded shredded Cheddar cheese, nitrous acid mMRS recovered significantly higher numbers of Lactobacillus delbrueckii ssp. bulgaricus versus traditional MRS. This higher sensitivity is relevant to the food industry because nitrous acid mMRS could increase hygiene monitoring of starter cultures by its ability to better detect and recover these or other relevant LAB from cheese. A recent study by Nwamaioha and Ibrahim (2018) showed that supplementing reinforced clostridial medium with $0.025 \% \mathrm{CaCl}_{2}, 0.01 \%$ uracil, and $0.2 \%$ Tween 80 significantly enhanced the growth rate of $L$. bulgaricus strains as measured by OD following $12 \mathrm{~h}$ incubation at $42^{\circ} \mathrm{C}$. Our results using $\mathrm{mMRS}$ expand the options beyond this important LAB species and also indicate that nitrous acid mMRS can recover higher counts of naturally occurring bacteria in cheese, which could also improve routine quality assurance/quality control by increasing the ability to detect and enumerate intended starter culture bacteria in cheese during cheesemaking, ripening, or both. This improved recovery would allow the dairy industry to monitor starter cultures for purity regarding phage infection (Jarvis, 1989) or presence of nonstarter bacteria that can alter the flavoring or contribute undesirable gas production to the cheese (Ortakci et al., 2015). Additionally, nitrous acid mMRS could be used for better culturing as cheese has shown to be a preferred vehicle for delivering LAB (Oberg et al., 2011). Davidson and Cronin (1973) employed nitrous acid and polymyxin B in for recovery of various lactic acid and enteric bacteria from a variety of foods. However, this study did not stipulate how the foods were seeded with these bacteria, at what initial densities (or whether these were naturally contaminated foods), nor which bacterial strains were being detected and in what specific foods. The authors of this study 

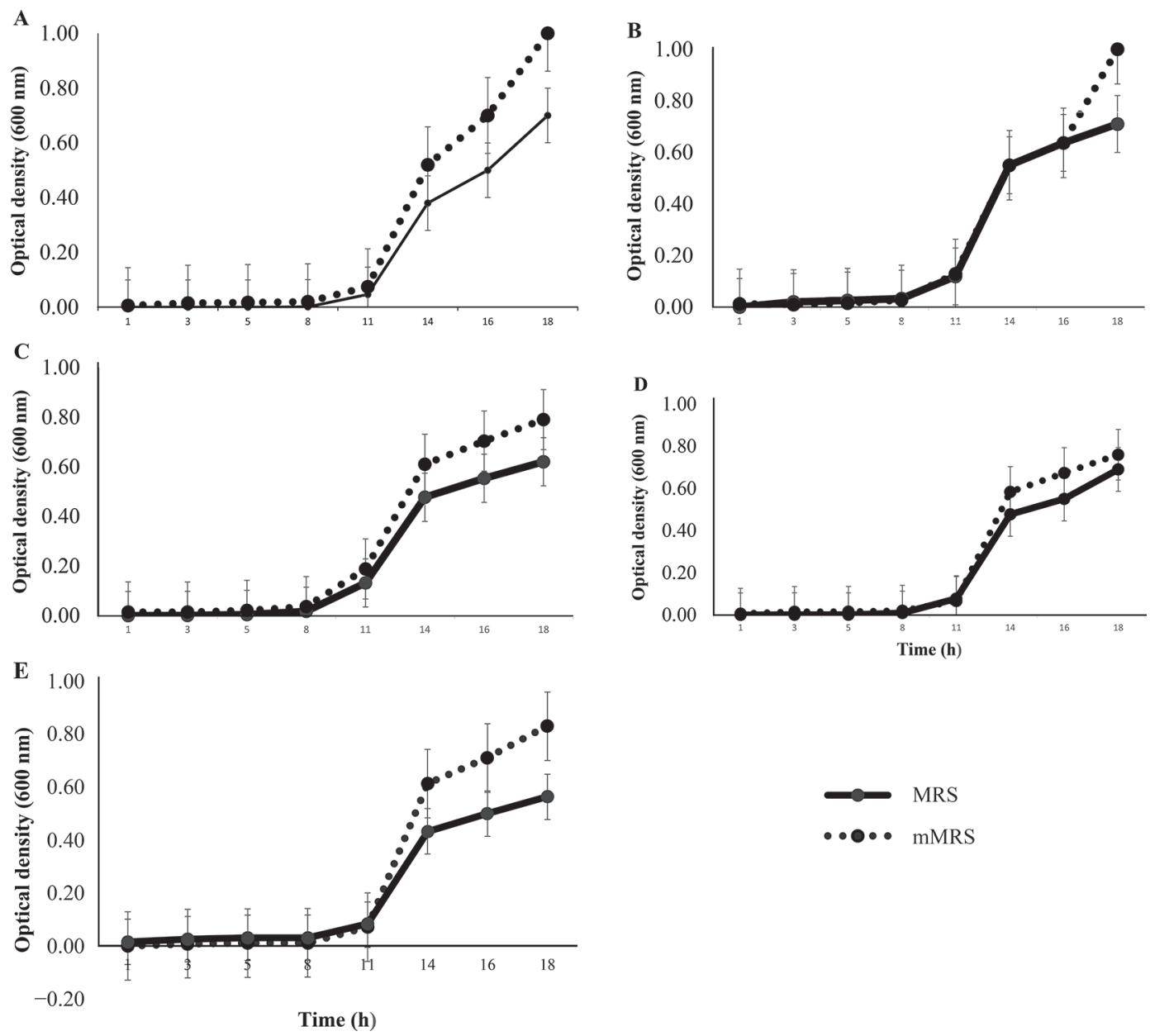

Figure 3. (A) Growth rate of Lactococcus lactis in modified de Man, Rogosa, and Sharpe (mMRS) was significantly higher by the $18 \mathrm{~h}$ time point compared with traditional de Man, Rogosa, and Sharpe (MRS) broth $(P<0.05)$. (B) Growth rate of Streptococcus salivarius in mMRS was significantly higher by the 18 -h time point compared with traditional MRS broth $(P<0.05)$. (C) Growth rate of Bifidobacterium longum in mMRS was significantly higher by the 18-h time point compared with traditional MRS broth $(P<0.05)$. (D) Growth rate of Lactobacillus delbrueckii ssp. bulgaricus in mMRS was significantly higher by the 18 -h time point compared with traditional MRS broth $(P<0.05)$. (E) Growth rate of Lactobacillus acidophilus in mMRS was significantly higher by the 18 -h time point compared with traditional MRS broth $(P<0.05)$.

also relied on polymyxin B as an added selective agent, which we omitted in our current study using modified MRS. Nitrous acid mMRS would be an improved medium to use for culturing and testing of these probiotic or LAB in cheesemaking.

Although we were not able to measure a significant increase in recovery rate of our LAB from seeded yogurt samples, we might suggest that this could be due to the heightened fastidious nature of many relevant yogurt bacteria, including $S$. thermophilus, which tends to be recovered more effectively on ST agar (Asraf and Shah, 2011), lactic agar, or M17 agar (Richter and Vedamuthu, 2001), using pour plates (preferred), rather than MRS medium in most procedures. A review on recovery of yogurt bacteria by Ashraf and Shah (2011) indicated that selective enumeration of $L$. acidophilus from a mixed yogurt culture could be made in Rogosa agar supplemented with 5-bromo-4-chloro-3-indolyl- $\beta$ D-glucopyranoside or with MRS agar containing maltose and incubation in a $20 \% \mathrm{CO}_{2}$ atmosphere. Alternatively, this same review suggested that Bifidobacterium spp. could be enumerated on MRS agar supplemented with nalidixic acid, paromomycin, neomycin sulfate, and lithium chloride under anaerobic incubation at $37^{\circ} \mathrm{C}$ for $72 \mathrm{~h}$; thus, efficient and reliable recovery of these yogurt bacteria tends to be best done on a genusby-genus basis rather than casting a wider net using a single medium for multiple LAB.

The selectivity evaluation of nitrous acid mMRS demonstrated that our tested LAB species grew to significantly higher densities in mMRS broth more rapidly than MRS. The mMRS broth supported higher overall OD values for most of the tested LAB versus plate-based mMRS, the specific reasons for this are not 


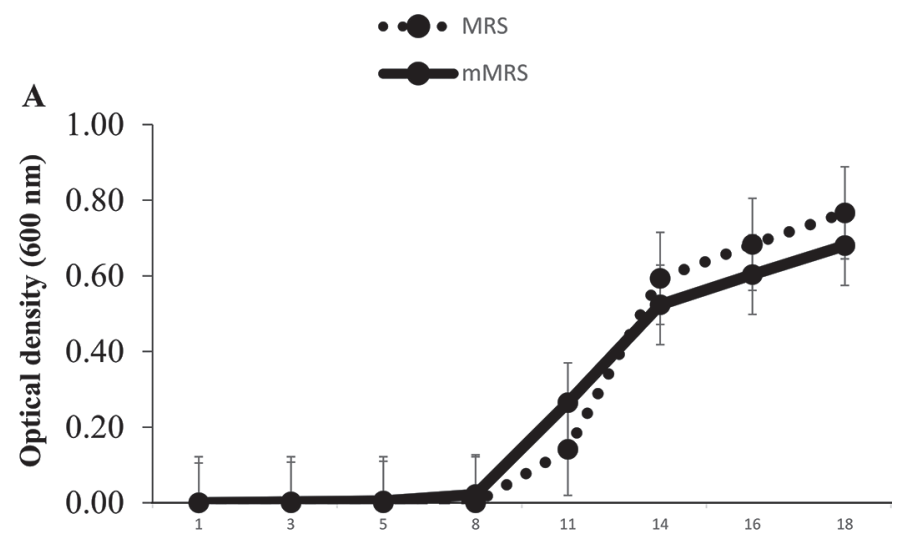

B

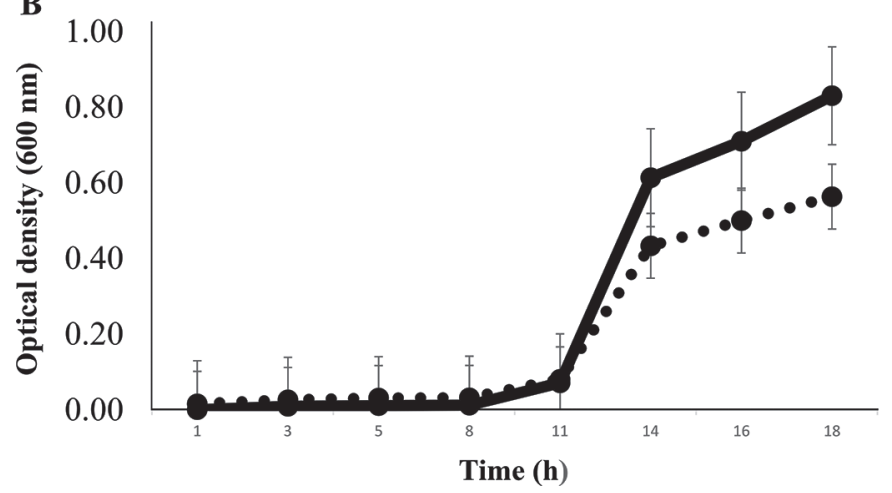

Figure 4. (A) Growth rate of Bacillus cereus ATCC14579 in modified de Man, Rogosa, and Sharpe (mMRS) was not significantly different during the 18-h incubation time compared with traditional de Man, Rogosa, and Sharpe (MRS) broth $(P>0.05)$. (B) Growth rate of Enterococcus faecalis in traditional MRS broth was significantly higher by the 18-h time point compared with mMRS broth $(P<0.05$ at $18 \mathrm{~h}$ ).

entirely evident but are subject to further investigation. Perhaps the plate-based recovery time point immediately preceded the period in the bacterial growth curve when densities markedly increased, as seen in the growth rate of mMRS broth curves, as most of the effect we observed in mMRS came quite close to the end of the time course of these experiments. Overall, these data reveal that nitrous acid supplemented mMRS is selective against at least one nontarget streptococcal species (E. faecalis), which tended grew significantly better in traditional MRS, while supporting higher growth rate of LAB commonly used for starter cultures and fermentation processes relevant to the dairy industry. This significantly higher growth in nitrous acid mMRS broth as compared with traditional MRS broth may have not be reflected in other food systems (for example, buttermilk), but our present study suggests that addition of a fundamental ingredient does offer great promise as a means to improve the utility of standard MRS broth or agar. The increased growth of LAB in nitrous acid mMRS could increase the hygiene and purity of starter cultures as LAB have shown to inhibit growth of pathogens or nonstarters that can contaminate cultures; therefore, the LAB growing faster to higher densities would be better able to outcompete these contaminants (Gutiérrez et al., 2016).

Sodium nitrite has long demonstrated bacteriostatic properties with the original mechanism based on the reactivity of sodium nitrite (Castellani and Niven, 1955). The bacteriostatic mechanism of sodium nitrite revolves around nitric oxide, the decomposition product of sodium nitrite. Nitric oxide is a small uncharged nonpolar molecule that can readily enter cells and is very reactive (Fang, 1997). Nitric oxide freely interacts with many nitrogen and oxygen intermediates within the cell to affect growth in complex pathways. Because nitric oxide is so reactive, it reacts with a wide range of cellular intermediates to form many different molecules affecting the cell in different ways. One particular nitric oxide molecule is peroxynitrite, which is formed through the interaction of nitric oxide and superoxide, commonly forced together in the presence of nitrous acid and hydrogen peroxide. Peroxynitrite is a powerful oxidant that reacts with many intermediates in the redox pathways within a cell, which greatly interferes with respiration as an aerobic growth mechanism, thus limiting the growth of microbes. Peroxynitrite is just one of the possible radicals that nitric oxide can form, but the radicals all operate through reactions with intermediates in cellular processes. Radicals can have bacteriocidal effects as they can react with amine, thiol, iron, and other groups of proteins. Nitric oxide radicals also affect DNA by deaminating DNA that interferes with DNA repair (Fang, 1997). Using these examples, it is easy to understand how a free radical like nitric oxide can hinder growth in such a wide range of ways.

Multiple studies have demonstrated the bacteriostatic effect of sodium nitrite on Listeria monocytogenes by extending lag phase and depressing growth (Junttila et al., 1989; Buchanan and Phillips, 1990). It is possible that this bacteriostatic action of sodium nitrite is the mechanism for the depressed growth of Enterococcus faecalis in nitrous acid mMRS. It has also been demonstrated that LAB have been associated with sodium nitrite depletion as these bacteria internalize sodium nitrite and metabolize it before it converts into more harmful forms, especially Leuconostoc mesenteroides (Oh et al., 2004). Therefore, nitrous acid mMRS can be more selective against nonstarters and contaminants of starter cultures by slowing their growth through the bacteriostatic action of sodium nitrite, whereas LAB will not be hindered and will grow to higher densities, thus outcompeting the other contaminants. It is possible that the other contaminant bacteria examined also grew to higher densities because they are related to 
LAB and could also deplete sodium nitrite; therefore, more species and strains could be examined in future work to conclude if relatives of LAB can deplete sodium nitrite. These mechanisms show the potential of nitrous acid mMRS as an alternative medium to culture and monitor the hygiene of LAB commonly used as starter cultures.

\section{ACKNOWLEDGMENTS}

The authors are grateful for partial support from the Ball State University Training Future Scientists (TFS) Program (author RR-H) and the Indiana STEM Louis Stokes Alliance for Minority Participation (IN LSAMP, to author NA), as well as technical assistance from Angie Boyle (Ball State University, Muncie, IN).

\section{REFERENCES}

Ao, X., X. Zhang, X. Zhang, L. Shi, K. Zhao, J. Yu, L. Dong, Y. Cao, and Y. Cai. 2012. Identification of lactic acid bacteria in traditional fermented yak milk and evaluation of their application in fermented milk products. J. Dairy Sci. 95:1073-1084.

Ashraf, R., and N. P. Shah. 2011. Selective and differential enumerations of Lactobacillus delbrueckii ssp. bulgaricus, Streptococcus thermophilus, Lactobacillus acidophilus, Lactobacillus casei and Bifidobacterium spp. in yoghurt-A review. Int. J. Food Microbiol. 149:194-208.

Biede, S. L., G. W. Reinbold, and E. G. Hammond. 1975. Influence of Lactobacillus bulgaricus on microbiology and chemistry of Swiss cheese. J. Dairy Sci. 59:854-858.

Buchanan, R. L., and J. G. Phillips. 1990. Response surface model for predicting the effects of temperature $\mathrm{pH}$, sodium chloride content, sodium nitrite concentration and atmosphere on the growth of Listeria monocytogenes. J. Food Prot. 53:370-376.

Carr, F. J., D. Chill, and N. Malada. 2002. The lactic acid bacteria: A literature survey. Crit. Rev. Microbiol. 28:281-370.

Castellani, A. G., and C. F. Niven Jr.. 1955. Factors affecting the bacteriostatic action of sodium nitrite. Appl. Microbiol. 3:154-159.

Collins, E. B. 1962. Behavior and use of lactic streptococci and their bacteriophages. J. Dairy Sci. 45:552-558.

Dave, R. I., and N. P. Shah. 1996. Evaluation of media for selective enumeration of Streptococcus thermophilus, Lactobacillus delbrueckii ssp. bulgaricus, Lactobacillus acidophilus, and bifidobacteria. J. Dairy Sci. 79:1529-1536.

Davidson, C. M., and F. Cronin. 1973. Medium for the selective enumeration of lactic acid bacteria from foods. Appl. Microbiol. 26:439-440.

Duncan, S. E., B. R. Yaun, S. S. Sumner, and J. Bruhn. 2004. Tech. comm., microbiological methods for dairy products. In Standard
Methods for the Examination of Dairy Products. 17th ed. H. M. Wehr and J. F. Frank, ed. American Public Health Association, Washington, DC.

Fang, F. C. 1997. Mechanisms of nitric oxide related antimicrobial activity. J. Clin. Invest. 99:2818-2825.

Gezginc, Y., F. Topcal, S. Comertpay, and I. Akyol. 2015. Quantitative analysis of the lactic acid and acetaldehyde produced by Streptococcus thermophilus and Lactobacillus bulgaricus strains isolated from traditional Turkish yogurts using HPLC. J. Dairy Sci. 98:1426-1434

Gutiérrez, S., H. Martínez-Blanco, L. B. Rodríguez-Aparicio, and M. A. Ferrero. 2016. Effect of fermented broth from lactic acid bacteria on pathogenic bacteria proliferation. J. Dairy Sci. 99:26542665.

Herve-Jimenez, L., I. Guillouard, E. Guedon, S. Boudebbouze, P. Hols, V. Monnet, E. Manguin, and F. Rul. 2009. Proteogenomic analysis of Streptococcus thermophilus cocultivated in milk with Lactobacillus delbrueckii ssp. bulgaricus: Involvement of nitrogen, purine, and iron metabolism. Appl. Environ. Microbiol. 75:2062-2073.

Jarvis, A. W. 1989. Bacteriophages of lactic acid bacteria. J. Dairy Sci. 72:3406-3428.

Junttila, J., J. Hirn, P. Hill, and E. Nurmi. 1989. Effect of different levels of nitrite and nitrate on the survival of Listeria monocytogenes during the manufacture of fermented sausage. J. Food Prot. $52: 158-161$.

Nwamaioha, N. O., and S. A. Ibrahim. 2018. A selective medium for enumeration and differentiation of Lactobacillus delbrueckii ssp. bulgaricus. J. Dairy Sci. 101:4953-4961.

Oberg, C. J., L. V. Moyes, M. J. Domek, C. Brothersen, and D. J. McMahon. 2011. Survival of probiotic adjunct cultures in cheese and challenges in their enumeration using selective media. J. Dairy Sci. 94:2220-2230.

Oh, C. K., M. C. Oh, and S. H. Kim. 2004. The depletion of sodium nitrite by lactic acid bacteria isolated from kimchi. J. Med. Food $7: 38-44$.

Ortakci, F., J. R. Broadbent, C. J. Oberg, and D. J. McMahon. 2015. Growth and gas production of a novel obligatory heterofermentative Cheddar cheese nonstarter lactobacilli species on ribose and galactose. J. Dairy Sci. 98:3645-3654.

Richter, R. L., and E. R. Vedamuthu. 2001. Milk and milk products. Pages 483-495 in Compendium of Methods for the Microbiological Examination of Foods. 4th ed. F. P. Downes and K. Ito, ed. American Public Health Association, Washington, DC.

Sawant, A. A., S. R. Pillai, and B. M. Jayarao. 2002. Evaluation of five selective media for isolation of catalase-negative Gram-positive cocci from bulk tank milk. J. Dairy Sci. 85:1127-1132.

Sharpe, M. E. 1979. Lactic acid bacteria in the dairy industry. J. Soc. Dairy Technol. 32:9-18.

\section{ORCIDS}

Matthew A. Renschler ( ) https://orcid.org/0000-0001-9631-7851 John L. McKillip ํ https://orcid.org/0000-0001-6369-7333 